\title{
The Rate and the Origin of Type Ia SNe in Radio Galaxies
}

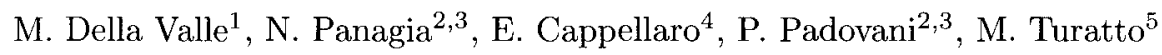

${ }^{1}$ INAF-Arcetri Astrophysical Observatory, Largo E. Fermi 5, 50125, Firenze, Italy; massimo@arcetri.astro.it

2 Space Telescope Science Institute, 3700 San Martin Drive, Baltimore, MD 21218, USA; panagia@stsci.edu; padovani@stsci.edu

3 Affiliated with the Space Telescope Division of the European Space Agency, ESTEC, Noordwijk, Netherlands

${ }^{4}$ INAF-Napoli, Salita Moiariello, 16, 80131, Napoli, Italy; cappellaro@na.astro.it

${ }^{5}$ INAF-Padova, Vicolo dell'Osservatorio 5; 35122, Padova, Italy; turatto@pd.astro.it

Summary. An analysis of type Ia supernova (SNIa) events in early type galaxies from the Cappellaro et al. [6] database provides strong evidence that the rate of type Ia supernovae ( $\mathrm{SNe}$ ) in radio-loud galaxies is about 4 times higher than the rate measured in radio-quiet galaxies, i.e. SNIa-rate(radio-loud) $=0.41_{-0.14}^{+0.13} h_{75}^{2} \mathrm{SNe}$ per century and per $10^{10} \mathrm{~L}_{\odot}^{B}$ (SNU) as compared to SNIa-rate(radio-quiet) $=0.11 \pm$ $0.03 h_{75}^{2}$ SNU. The exact value of the enhancement is still rather uncertain, but is likely to be in the range $\sim 2-7$. We discuss the possible causes of this result and we conclude that the enhancement of the SNIa explosion rate in radio-loud galaxies has the same common origin as their being strong radio sources, but that there is no causality link between the two phenomena. We argue that repeated episodes of interaction and/or mergers of early type galaxies with dwarf companions are responsible for inducing both strong radio activity in $\sim 14 \%$ of early type galaxies, and the $\sim 1$ Gyr old stellar population needed to supply an adequate number SNIa progenitors.

\section{Introduction}

The knowledge of the supernova rate is essential for constraining the mechanisms of galaxy formation and for understanding galaxy evolution. Particularly the chemical evolution of systems that at the present epoch have a reduced or totally exhausted star formation activity, such as the elliptical galaxies, dramatically depends on the rate of type Ia events (e.g. [18]). Thus, any mechanism capable of producing an enhancement of SNIa rate in this type of galaxies should be regarded with the highest interest. Two recent works $[4,12]$ deal with this possibility. Capetti $[4]$ finds a close association between the position of 6 type Ia $\mathrm{SNe}$ discovered in radio galaxies and their jets, while [12] claim the detection of an enhancement of the nova rate in 
M87 in the regions near its jet. The former result would indicate that type Ia $\mathrm{SNe}$ in radio galaxies show a statistically significant preference (at the level of $\sim 95 \%$ ) to occur close to their jets. The latter result may suggest that the presence of jets could increase the accretion rate from the interstellar medium onto the white dwarfs by 1-2 orders of magnitude, up to $\sim 10^{-8}-10^{-9} \mathrm{M}_{\odot}$ $\mathrm{yr}^{-1}$, which is the rate necessary to trigger classical nova explosions [17]. By combining the two results, one would infer that the presence of the jets may increase the efficiency of accretion rate from the ISM and drive an accreting white dwarf to approach the Chandrasekhar mass and ignite, according to the standard single-degenerate model, a SNIa explosion ([12], and references therein). In this scenario it is the jet itself that alters the physical conditions of its environment and thus it is the direct cause of a local enhancement of nova and type Ia SN explosions.

Since jets are outstanding signatures of radio galaxies (e.g. [3]), it is important to extend the investigation to all galaxies with strong radio emission and to study the SNIa rate as a function of the radio activity. In this paper we use a larger SN databases to extend the study of [8] to investigate if, and to which extent radio galaxies exhibit an over-production of type Ia SNe with respect to quiescent galaxies of the same luminosity class, and to clarify what is the dominant mechanism that drives this process. In the following, we will denote as radio-loud sources, or radio galaxies, all galaxies which have radio luminosities at $1.4 \mathrm{GHz}$ higher than $10^{29} \mathrm{erg} \mathrm{s}^{-1} \mathrm{~Hz}^{-1}$, and $\mathrm{M}_{R}<-20.5$ (e.g. $[11,19])$ and radio-quiet all galaxies with radio luminosities below that threshold. The threshold value of $\sim 10^{29} \mathrm{erg} s^{-1} \mathrm{~Hz}^{-1}$ is the faint limit of the luminosity function for the radio-galaxies (see also [20]), and, therefore, it represents the most natural threshold to identify "radio-loud" galaxies.

\section{Statistics of SNIa Explosions in Early Type Galaxies}

Among the several databases on supernovae currently available, we have used the one compiled by Cappellaro, Evans and Turatto [6] which has the advantage of being defined a priori, i.e. the galaxies to be monitored were selected before the SN explosions. As a consequence our statistical analysis does not suffer of any kind of a posteriori selection criteria, which actually may affect the statistical analysis carried out on compilations of parent galaxies of SNe. An inspection to Table 1 reveals the following facts:

1. The fraction of radio galaxies in our sample is 0.12 of the whole sample of galaxies. Such fraction is marginally smaller than $\sim 0.14$ reported by [11] for radio galaxies having the same radio luminosities $\left(>10^{29} \mathrm{erg} \mathrm{s}^{-1}\right.$ $\mathrm{Hz}^{-1}$ at $1.4 \mathrm{GHz}$ ). This indicates that the frequency of radio galaxies in our sample is not affected by biases related to their radio properties.

2. Separating radio-loud from radio-quiet galaxies, we have 9 SNIa events in radio-loud galaxies and 10 in radio-quiet galaxies. Therefore, we can 
Table 1. Number of Galaxies, Surveillance time $T\left(\mathrm{yr} \times \mathrm{L}^{B} / 10^{10} \mathrm{~L}_{\odot}^{B}\right)$ and Number of SNIa events

\begin{tabular}{lrrr}
\hline \multicolumn{1}{c}{ Galaxies } & $N$ & $T$ & SNe \\
\hline radio-quiet & 2216 & 8894 & 10 \\
radio-loud & 274 & 2220 & 9 \\
total & 2490 & 11114 & 19 \\
\hline
\end{tabular}

confidently estimate the rate of type Ia SNe for radio-loud galaxies to be $0.41_{-0.14}^{+0.13} h_{75}^{2}$ SNU (the errors are estimated from a direct application of Poisson statistics), whereas the rate of type Ia SNe in radio-quiet systems turn out to be $0.11 \pm 0.03 h_{75}^{2} \mathrm{SNU}$.

\section{Discussion and Conclusions}

An analysis of [6] database provides strong evidence that the rate of type Ia $\mathrm{SNe}$ in radio galaxies is about 4 times higher than the rate measured in quiescent galaxies. The actual value of the enhancement is still rather uncertain, due to the uncertainties involved in the SN rate measurements (see [5]) but is likely to be in the range $\sim 2-7$. This confirms the result recently obtained by [8] on the basis of a smaller statistical sample. The observed enhancement of the rate of SNIa events in radio galaxies can be explained in basically two different ways:

1. The enhanced SNIa explosion rate in radio-loud galaxies is a direct consequence of their being strong radio sources. This is the scenario suggested by [4] and [12]. In radio galaxies the accretion rate from the interstellar medium onto white dwarfs could be enhanced by the action of the jets. This process could also account for the high rate of novae observed in M87 in the regions nearby the jets. The association between type Ia SNe and jets requires a further step, i.e. that the progenitors of these SNeIa are cataclysmic-type systems, according to the single degenerate scenario. In this hypothesis, the enhancement of SNIa in radio galaxies is expected to be spatially confined to the regions immediately adjacent to radio jets and/or the bulk of radio activity. While this may be true for Virgo A (M87), it appears not to be the case for Fornax A (NGC 1316), in which the two SNIa (SN 1980N and SN 1981D) are located quite far from strong radio lobes ([9]), nor for Centaurus A (NGC 5128), in which SN 1986G is deeply embedded in the equatorial lane of gas and dust. At any rate, if the dominant phenomenon is accretion from the ISM rather than from a binary companion, an extreme consequence would be that single WDs will contribute to Nova and perhaps SNIa production, and not only binary systems with suitable parameters. 
Actually, since the fraction of binary systems that may give rise to a SNIa is only $5-10 \%$ of the total number of systems with masses above 3 solar masses [13], one should expect a very dramatic increase of the SNIa rate in jet dominated radio-galaxies, by factors as high as 20 or higher if direct accretion from ISM becomes the dominant accretion process.

2. The enhancement of SNIa explosion rate in radio-loud galaxies has the same common origin as their being strong radio sources, but there is no causality link between the two phenomena. We favor this alternative. Indeed, the radio activity of a galaxy is most likely triggered by interaction and/or mergers (e.g. $[1,2,10]$ ). Also, the SNIa rate can be enhanced by the formation or the capture of relatively young stellar populations in which SNIa occur at much higher rates than in genuinely old populations. SNIa progenitors are stars with original masses above $\sim 3$ solar masses, having nuclear-burning lifetimes shorter than $\sim 400$ Myrs. After that time, the star becomes a white dwarf and may explode as a SNIa only after an additional time as needed for either accreting mass from a companion or merging with it. Such a time is essentially unconstrained by theory, but measurements of SNIa rates at different redshifts suggest that it should be shorter than $\sim 1$ Gyrs (e.g. $[13,15])$. As a consequence, in order to sustain a substantial rate of SNIa explosion, a galaxy has to have a steady supply of young stellar populations, at least over a time-scale of 1-2 Gyrs. In late type galaxies, the active star formation provides the needed input for a steady production of SNIa events. Such a supply in early type galaxies is naturally provided by repeated episodes of interaction or mergers that induces either formation of young stellar populations (galaxy interaction; e.g. the Antennae galaxies [21]) or capture of young stars from dwarf companions. Well known examples of interacting and/or merging early type galaxies are Centaurus A (NGC 5128) and Fornax A (NGC 1316), which are strong radio sources and indeed have produced $1+2 \mathrm{SNIa}$ in the last century. Moreover, the Virgo Cluster radio galaxy NGC 4753, had given birth to another SNIa (SN1965I) less than two decades before, thus confirming its high SNIa rate.

Also in favor of this hypothesis is the fact that the observed SNIa rates are higher in late type galaxies than in early type galaxies (about a factor of 10 between Ellipticals and Sd galaxies) once the rates are normalized to the galaxy $\mathrm{H}$ or $\mathrm{K}$ band luminosities, and, therefore, to their masses in stars, rather than to their B-band magnitudes $[7,14,16]$. This fact confirms the connection between recent stellar populations and type Ia supernovae, and provides a natural explanation of why among early type galaxies are SNIa rates higher in radio galaxies, without requiring the concurrence of any new and/or additional process to enhance the SNIa rates.

Finally, we note that in our scenario the occurrence of a few type II$\mathrm{Ib} / \mathrm{c}$ SNe in early type galaxies is expected at the early stages of the same 
star formation burst that provides a steady supply of SNIa events. Since core collapse $\mathrm{SNe}$ are produced by progenitors more massive than $8 \mathrm{M}_{\odot}$, whose lifetime is shorter than about 30 Myrs, only for a small fraction of the time between subsequent interaction/capture episodes (approximately $30 \mathrm{Myrs} / 1 \mathrm{Gyr}=3 \%$ ) would one expect to see such SNe in early type galaxies. Since in galaxies with active star formation the rate of core collapse SNe is about 4 times higher than that of SNIa, we estimate that one should expect to detect core collapse $\mathrm{SNe}$ at an average rate of about $0.03 \times 4 \simeq 12 \%$ that of SNIa events. Since 9 SNIa were discovered in E/SO galaxies during time-controlled surveys ([5]), one would expect the occurrence of about $1 \pm 1$ core collapse event in those galaxies, but none has been detected so far. It is then clear that, while missing one event is statistically acceptable within the currently available statistics, our prediction will be observationally testable when the SNIa statistics will have increased appreciably.

\section{References}

1. W. Baade, R. Minkowski: Astrophys. J. 119, 206 (1954)

2. B. Balick, T.M. Heckman: Ann. Rev. Astron. Astrophys. 20, 431 (1982)

3. G.V. Bicknell: New Astron. Rev. 46, 365 (2002)

4. A. Capetti: Astrophys. J. Lett. 574, L25 (2002)

5. E. Cappellaro, M. Turatto, S. Benetti, D.Yu. Tsvetkov, O.S. Bartunov, I.N. Makarova: Astron. Astrophys. 268, 472 (1993)

6. E. Cappellaro, R. Evans, M. Turatto: Astron. Astrophys. 351, 459 (1999)

7. M. Della Valle, M. Livio: Astrophys. J. Lett. 423, L31 (1994)

8. M. Della Valle, N. Panagia: Astrophys. J. Lett. 587, L71 (2003)

9. B.J. Geldzahler, E.B. Fomalont: Astron. J. 89, 1650 (1984)

10. T.M. Heckman et al. : Astrophys. J. 311, 526 (1986)

11. M.J. Ledlow, F.N. Owen: Astron. J. 112, 9 (1996)

12. M. Livio, A. Riess, W. Sparks: Astrophys. J. Lett. 571, L99 (2002)

13. P. Madau, M. Della Valle, N. Panagia: Mon. Not. R. Astron. Soc. 297, L17 (1998)

14. F. Mannucci, R. Maiolino, G. Cresci, M. Della Valle, L. Vanzi, F. Ghinassi, V.D. Ivanov, N.M. Nagar, A. Alonso-Herrero: Astron. Astrophys. 401, 519 (2003)

15. R. Pain et al. : Astrophys. J. 577, 120 (2002)

16. N. Panagia: In: Experimental Physics of Gravitational Waves. eds. G. Calamai, M. Mazzoni, R. Stanga, F. Vetrano (World Scientific: Singapore, 2002) pp. 107119

17. T. Prialnik, A. Kovetz: Astrophys. J. 445, 789 (1995)

18. A. Renzini, L. Ciotti, A. D'Ercole, S. Pellegrini: Astrophys. J. 419, 52 (1993)

19. E.M. Sadler, C.R. Jenkins, C.G. Kotanyi: Mon. Not. R. Astron. Soc. 240, $591(1989)$

20. C.M. Urry, P. Padovani: Pub. Astron. Soc. Pacific 107, 803 (1995)

21. B.C. Whitmore, Q. Zhang, C. Leitherer, S.M. Fall, F. Schweizer, B.W. Miller: Astron. J. 118, 1551 (1999) 